Www.jmscr.igmpublication.org

Index Copernicus Value: 79.54

ISSN (e)-2347-176x ISSN (p) 2455-0450

crossrefDOI: https://dx.doi.org/10.18535/jmscr/v7i2.90

\title{
Osteoporosis in Chronic Obstructive Pulmonary Disease Patients - A Hospital Based Cross-Sectional Study
}

\author{
Authors \\ Dr Sophia Philip*, Dr Juliet John, Dr Varghese M.T, Dr Manjula V.D \\ Department of Respiratory Medicine, Government Medical College, Ernakulam, Kerala \\ *Corresponding Author \\ Dr Sophia Philip \\ 31/1179 A 1, Sahakarna Road, Ponnurunni, Vyttila, Kochi Kerala. 682019, India \\ Mob: 9447562941, Email: sophiaphilip@ rediffmail.com
}

\begin{abstract}
COPD is a syndrome of progressive airway limitation caused by the abnormal inflammatory reaction of the airways and lung parenchyma. Osteoporosis is one the major extra pulmonary manifestations of COPD. Osteoporosis is, characterized by a decrease in bone mineral density (BMD) \& or deterioration of microarchitecture resulting in increase bone fragility \& hence an increased susceptibility to fractures.

A cross-sectional study was conducted in a tertiary care centre to find the proportion of patients with osteoporosis among COPD patients using Calcaneal BMD estimation \& X-Ray lumbosacral spine.

172 COPD patients were subjected to Calcaneal Quantitative Ultrasound scan \& X-Ray lumbosacral spine \& evaluated for signs of osteoporosis.

Out of the 172 patients studied 90 (52.3\%) patients had osteoporosis with estimation of BMD \& X-Ray spine \& osteopenia in $64(37.2 \%)$ \& $18(10.5 \%)$ were detected to be normal with BMD estimation. With X-Ray sine $57(33.1 \%)$ were osteopenic \& $25(14.5 \%)$ were normal..

Thus a significant number of patients were detected to be osteoporotic with BMD \& X-Ray screening, \& also $27 \%$ of the patients had fracture vertebra, without any history of obvious injury. There was also significant negative co-relation seen with BMI in both BMD \& X-Ray.

As the stage \& duration of COPD advanced, significant number of patients developed osteoporosis. COPD patients are prone to osteoporosis due to their low BMI, impaired mobility \& co-morbidities.

Keywords: COPD, BMD, Calcaneal QUS.
\end{abstract}

\section{Introduction}

Chronic Obstructive pulmonary disease (COPD) is a chronic inflammatory lung disease that causes obstructed airflows from the lungs. Symptoms include breathing difficulty, cough, mucus production, \& wheezing, caused by long term exposure to irritating gases or particulate matter, most often from cigarette smoke. People with
COPD are at risk of developing heart diseases, lung carcinoma \& variety of other conditions.

COPD has significant extra pulmonary effects the so called systemic effects of COPD. Weight loss, nutritional abnormalities, skeletal muscle dysfunction are well recognized systemic effects of COPD. Other less well known but potentially important systemic effects include an increased 
risk of cardiovascular disease \& several neurologic \& skeletal defects. The mechanisms underlying these systemic effects are unclear, but they are probably inter- related \& multifactorial including inactivity, systemic inflammation, tissue hypoxia $\&$ oxidative stress ${ }^{[1]}$. The skeletal muscle dysfunction is common, due to under use \& reduction in body weight, in response to increased systemic inflammatory mediators. ${ }^{[1]}$

Other potential origins of systemic inflammation in COPD include smoking, lung hyperinflation, tissue hypoxia, skeletal muscle dysfunction \& bone the bone marrow. ${ }^{[2]}$

Osteoporosis and its related fractures are common $\&$ have significant impacts on quality of life \& even respiratory function in patients with COPD. COPD associated osteoporosis is however extremely under treated. Recent studies have suggested that both reduced bone mineral density (BMD) \& impaired bone quality contribute to bone fragility, causing fractures in COPD patients. ${ }^{[3]}$. COPD significantly increased the risk of osteoporosis (low BMD) 1.9 fold, although COPD subjects were 2.7 years older $\&$ included $7.1 \%$ more females, in one study. Most other cross sectional studies comparing COPD patients with controls \& population based cohort studies have also estimated the COPD associated risk of osteoporosis at approximately $1.5-2$ fold $^{[3]}$

Osteoporosis in COPD is multifactorial. Many risk factors have been involved in development of osteoporosis in COPD. Patients who have moderate to severe COPD have advanced nature of the disease which predisposes them to osteoporosis, by virtue of them being elderly, or chronically disabled \& having chronic systemic inflammation. Prevalence of systemic \& extra pulmonary manifestation in COPD is already proved in many studies. Patients with COPD are often treated with oral or parenteral glucocorticoids during exacerbations. Such oral or parenteral glucocorticoid therapy along with various other risk factors clearly increase the risk for development of osteoporosis ${ }^{[4]}$
Hence in an ideal set up all patients with COPD should be screened for osteoporosis using BMD measurements made by Dual Energy Absorptiometry (DEXA) scan, which is considered the Gold standard method for the early diagnosis. So that the proper therapy of this condition can be advised. This will help in improving the quality of life in these patients \& thus improving the morbidity of this condition ${ }^{[4]}$ WHO defines osteoporosis based upon DEXA measurements, it provides accurate measurements at clinically relavent sites. The major disadvantage of DEXA is non portability \& relatively expensive compared to alternative peripheral technologies \& the use of low dose radiation.

Alternative techniques for evaluation of bone status at peripheral sites have been developed. Among them Quantitative Ultrasound (QUS) measurements of the heel is considered one of the best alternative current method available for the assessment of fracture risk. Indeed it is portable, cheaper \& easier to perform in comparison with DEXA $^{.[5]}$

QUS technology provides additional information on bone micro-architecture as well as BMD. Several studies also indicated that it can predict fractures for both genders. An early detection would allow preventive measures to be taken to hinder the progression of osteoporosis ${ }^{[6]}$ Calcaneal ultrasound measurement was seen as useful tool in nursing home residents. ${ }^{[7]}$

However in developing countries with insufficient resources there is a practical problem of applying screening procedures to all COPD patients, with DEXA scan.

So this study was undertaken to detect osteoporosis in COPD patients with QUS \& XRay Lumbosacral spine, to create an awareness among the patients \& treating Physicians about this entity, so that an early treatment modality can be planned.

\section{Materials \& Methods}

This cross - sectional study was conducted on 172 COPD patients in a tertiary care, teaching 
centre. Study duration was for a period of eighteen months, from 2015 after obtaining clearance from Ethical Committee.

Male \& female patients between 40-65 years who were diagnosed with COPD after detailed history, physical examination \& relevant investigations as per department protocol were recruited \& classified as per GOLD staging.

Patients with Asthma overlap, Connective tissue disorders, or Interstitial Lung diseases on Chronic steroid therapy \& patients with malignancies were excluded from the study.

Data was collected using a structured questionnaire which consisted of sociodemographic variables, after obtaining informed consent.

Calcaneal quantitative Ultrasound \& X-Ray Lumbosacral spine was done for all patients , falling in the inclusion criteria. $T$ Score was estimated. Bone Mineral Density was estimated $\&$ categorized as Normal ( $\mathrm{T}$ score upto -1 ), Osteopenia ( $\mathrm{T}$ score -1 to -2.4 ), Osteoporosis ( $\mathrm{T}$ score $=/<-2.5$ )

X-Ray of Lumbosacral spine was analysed by a radiologist \& an orthopedician separately \& findings were reported as Normal, Osteopenia, \& evidence of osteoporosis was assessed with presence of cortical thinning, increased radiolucency or fracture of vertebra.

\section{Statistical Analysis}

Data was coded \& entered in Microsoft Excel \& analysis was done using SPSS version 21. Quantitative variables were summarized as frequency, \& percentage. 95\% Confidence Interval was estimated. Since there were more than 2 groups, ANNOVA test was used to find out the significant difference between means of $T$ Score in different stages of COPD. Chi square test was applied to find the association between qualitative variables.

Pearson Correlation quotient was used to find out the correlation between BMI of patient \& duration of COPD.

\section{Results}

Out of the 172 patients recruited for the study, 37 (21\%) were females. Mean age of the study population was 59.12 with SD 7.2. 54\% of patients were above 60 years of age, out of which $59.6 \%$ were osteoporotic, $35.1 \%$ had osteopenia \& 5.3\% had normal BMD. (Table1)

There was a significant difference in BMD \& XRay between age groups with ANOVA \& Chi square tests, which indicated that in COPD patients with advanced age the BMD significantly decreased \& X-Ray showed features of osteoporosis. $\mathrm{P}$ value being 0.002 \& 0.001 respectively.

\section{Relation with stages of COPD}

All patients were staged into Mild, Moderate, Severe \& Very severe, as per GOLD staging. This study showed that the number of patients with osteoporosis increased significantly as the stage of COPD advanced. By One way ANOVA test, $70.6 \%$ of patients with severe COPD had osteoporosis with BMD estimation \& evidence on $\mathrm{X}$-ray LS spine. P value being 0.000. (Table 1)

\section{Duration of COPD \& Smoking}

By One way ANNOVA \& Chi square test, it was found that, the number of patients having osteoporosis was more as the duration of COPD increased. $\mathrm{P}$ value $=0.000$. The mean duration of COPD with normal BMD 4.5 years, Osteopenia was 5.04 years \& Osteoporosis was 7.2 years. $34.6 \%$ of patients had osteoporosis when duration of COPD was < 5years, which increased to $85.7 \%$ when duration was $>10$ years when estimated with BMD as well as X-Ray spine. (Table 1)

A negative correlation was seen with duration of COPD \& BMD when Pearson Correlation test was applied. $\mathrm{r}$ is $-0.219, \mathrm{p}$ value 0.004

Out of the 172 patients, 42 (24\%) were non smokers, $29(16 \%)$ current smokers \& 101 (57.7\%) reformed smokers. $19 \%$ of non smokers, $29 \%$ of current smokers \& $56.4 \%$ of reformed smokers were detected to have osteoporosis. But significant association between smoking \& osteoporosis could not be established 
with ANOVA \& Chi square test as $\mathrm{P}$ value was 0.570 .

\section{BMI}

There was a negative correlation found between BMI \& osteoporosis with Pearson correlation testing ( $\mathrm{r}$ is 0.245 ). Mean BMI in COPD patients without osteoporosis was 22.08 where as in COPD with osteoporosis was 19.81. With ANOVA there was a significant association seen with BMI \& osteoporosis as P is 0.004 . So as BMI decreases, osteoporosis increases. (Table 4)

\section{T Score}

Mean T Score in patients with normal BMD was $-0.267 \&$ for Osteopenia was -1.98 \& osteoporosis was -2.74. Significant association of $\mathrm{T}$ score with stages of COPD was seen with ANOVA \& Chi Square in this study. $T$ score reduced as the stage of COPD advanced. $\mathrm{P}$ value $=0.000$. Mean T score in Mild COPD is -1.41 , Moderate COPD is -2.18 , Severe COPD is -2.43 $\&$ Very Severe is COPD is -2.52 . (Table 2)

\section{Co-Morbidities}

No significant association was found between osteoporosis \& Type II Diabetes Mellitus, P value is 0.458. But significant association was seen in cardiac disease \& osteoporosis, when estimated with BMD, $\mathrm{P}$ value $=0.043$. (Table 3$)$

Out of the 172 patients studied 25 patients were detected to be Normal by both X-Ray Spine \& BMD estimation. $64(37.2 \%)$ patients were detected to be osteopenic by BMD, but X-Ray could detect only 57 patients. 25 patients who were detected to be normal according to Xray spine had osteopenia according to BMD estimation.

Both the parameters detected 90 patients to have osteoporosis which suggests that BMD is more sensitive \& can be used as a screening tool. On analysis of X-Ray features $57.8 \%$ had cortical thinning , $13.3 \%$ had increased radiolucency, \& $27.8 \%$ had fracture Vertebra. $>20 \%$ had all the 3 features.

Table 1 : Association of osteoporosis with smoking status, Age group, stages \& duration of COPD

\begin{tabular}{|c|c|c|c|c|c|c|}
\hline \multirow{4}{*}{ 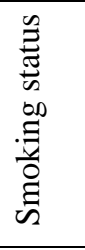 } & & $\mathrm{N}$ & NORMAL & OSTEOPENEA & OSTEOPOROSIS & $\mathrm{P}$ value \\
\hline & Non-smoker & $42(24.4 \%)$ & $6(14.3 \%)$ & $17(40.5 \%)$ & $19(45.2 \%)$ & \multirow{3}{*}{0.725} \\
\hline & $\begin{array}{l}\text { Current } \\
\text { Smoker }\end{array}$ & $29(16.86 \%)$ & $5(17.2 \%)$ & $10(34.5 \%)$ & $14(48.5 \%)$ & \\
\hline & $\begin{array}{l}\text { Reformed } \\
\text { smoker }\end{array}$ & 101(58.72\%) & $14(13.9 \%)$ & $30(29.7 \%)$ & $57(56.4 \%)$ & \\
\hline \multirow{3}{*}{ 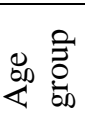 } & $40-50$ & $23(13.37 \%)$ & $6(26.1 \%)$ & $13(56.5 \%)$ & $4(17.4 \%)$ & \multirow{3}{*}{0.002} \\
\hline & $51-60$ & $55(31.97 \%)$ & $7(12.7 \%)$ & $18(32.7 \%)$ & $30(54.5 \%)$ & \\
\hline & $>60$ & $94(54.65 \%)$ & $5(5.3 \%)$ & $33(35.1 \%)$ & $56(59.6 \%)$ & \\
\hline$\overline{0}$ & Mild COPD & $39(22,67 \%)$ & $13(13.3 \%)$ & $23(59 \%)$ & $3(77 \%)$ & \multirow{4}{*}{0.000} \\
\hline \multirow{3}{*}{ 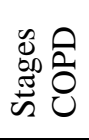 } & Mad COPD & $55(319 \%)$ & $4(73 \%)$ & $28(500 \%)$ & 234180 & \\
\hline & Severe COPD & $61(35.45 \%)$ & 0 & $9(14.8 \%)$ & $52(85.2 \%)$ & \\
\hline & V.Severe & $17(9.88 \%)$ & $1(5.9 \%)$ & $4(23.5 \%)$ & $12(70.6 \%)$ & \\
\hline \multirow{3}{*}{ ن } & $1-5$ & $81(47.09 \%)$ & $17(21 \%)$ & $36(44.4 \%)$ & $28(34.6 \%)$ & \multirow{3}{*}{0.000} \\
\hline & $6-10$ & $84(48.83 \%)$ & $7(8.3 \%)$ & $21(25 \%)$ & $56(66.7 \%)$ & \\
\hline & $>10$ & $7(4.06 \%)$ & 1 & 0 & $6(85.7 \%)$ & \\
\hline
\end{tabular}

Table 2: Descriptives of $\mathrm{T}$ score

\begin{tabular}{|l|c|c|c|}
\hline & Mean of T score & Std Deviation & Std error ofmean \\
\hline Normal & -0.267 & 0.676 & 0.159 \\
\hline Osteopenea & -1.98 & 0.358 & 0.044 \\
\hline Osteoporosis & -2.74 & 0.204 & 0.021 \\
\hline
\end{tabular}


Table 3 : Association of CARDIACDISEASE with BMD

\begin{tabular}{|c|c|c|c|c|c|c|c|}
\hline & \multicolumn{3}{|c|}{ RESULTBMD } & \multirow[t]{2}{*}{ Total } & \multirow{2}{*}{$\begin{array}{c}\mathrm{P} \\
\text { Value }\end{array}$} \\
\hline & & & NORMAL & OSTEOPENIA & OSTEOPOROSIS & & \\
\hline \multirow{5}{*}{ CARDIACDISEASE } & & Count & 4 & 15 & 37 & 56 & \multirow{7}{*}{0.043} \\
\hline & YES & of within & $71 \%$ & $268 \%$ & $661 \%$ & $1000 \%$ & \\
\hline & & CARDIACDISEASEYN & 1.110 & 20.010 & $00.1 / 0$ & 100.07 & \\
\hline & & Count & 14 & 49 & 53 & 116 & \\
\hline & NO & $\begin{array}{c}\% \text { within } \\
\text { CARDIACDISEASEYN }\end{array}$ & $12.1 \%$ & $42.2 \%$ & $45.7 \%$ & $100.0 \%$ & \\
\hline \multirow{2}{*}{\multicolumn{2}{|c|}{ Total }} & Count & 18 & 64 & 90 & 172 & \\
\hline & & $\begin{array}{c}\% \text { within } \\
\text { CARDIACDISEASEYN }\end{array}$ & $10.5 \%$ & $37.2 \%$ & $52.3 \%$ & $100.0 \%$ & \\
\hline
\end{tabular}

Table 4: Association of BMI with BMD

\begin{tabular}{|l|c|c|c|c|}
\hline & Mean BMI & Std Deviation & Std Error & P value \\
\hline Normal & 22.08 & 3.37 & 0.674 & \multirow{2}{*}{0.004} \\
\cline { 1 - 4 } Osteopenia & 21.63 & 4.22 & 0.560 & \\
\cline { 1 - 4 } Osteoporosis & 19.81 & 3.66 & 0.386 & \\
\hline
\end{tabular}

\section{Discussion}

It is already a known fact that COPD is a chronic inflammatory disease. COPD \& Osteoporosis are strongly associated because of common risk factors such as age, smoking \& inactivity. In addition COPD related systemic inflammation , Vitamin D deficiency \& the use of systemic corticosteroids during exacerbations enhance ongoing bone destruction. ${ }^{[8]}$

A study published in Egyptian journal of Chest diseases \& Tuberculosis in 2014 have showed a high prevalence of low Bone Mineral Density (56.6\%)among men with COPD than age \& sex matched healthy individuals \& the degree of the loss of BMD has been found to be proportionate to the severity of the disease. COPD patients with low BMD, have threefold increase fractures risk than COPD patients with normal BMD ${ }^{[11]}$

In this study BMD was assessed by heel Ultrasound attenuation bone densitometer because it is readily available \& easy to perform and XRay lumbo-sacral spine was studied for evidence of osteoporosis.

Though DEXA scan is the gold standard for assessing osteoporosis according to WHO, many studies have compared DEXA with Quantitative Ultrasound measurement of the heel. In one study it was seen that although DEXA was not replaceable by QUS, but can be incorporated in diagnostic strategy as triage test prior to DEXA to reduce the need for DEXA scans \& performs better than a risk score based on clinical parameters ${ }^{[9]}$, whereas in another study, there was a moderate correlation between $\mathrm{T}$ - Scores measured by DEXA \& by QUS ${ }^{[10]}$

Study by International Osteoporosis Foundation found that prevalence of osteoporosis rises from $28.6 \%$ in GOLD stage II to $75 \%$ in GOLD stage IV \& also found that patients in GOLD stage IV have $7.6 \%$ times greater risk of low BMD than patients in GOLD stage II ${ }^{[12]}$

Our study was also in line with this study where we found $70.6 \%$ of patients in stage IV COPD had osteoporosis.

Our study also found a strong association of low BMD with duration of COPD i.e $85.7 \%$ of patients who had features of COPD $>10$ years had osteoporosis. Also found that the BMD was low in patients who were in the age group of 50-65, than in patients who were between 40-50years. A similar result was seen in a study done in Maulana Azad Medical College, New Delhi where Stage II COPD patients had less number of osteoporotic patients than Stage III \& IV \& BMD was lower in age group 51-60, when compared to patients in age group of $41-50^{[13]}$ 
Smoking may also cause osteoporosis. A meta analysis by ward \& Klesges demonstrated that tobacco smoking had a cumulative, dose dependent independent effect on bone mass ${ }^{[15]}$. In our study the percentage of patients with osteoporosis were definitely more in smokers \& reformed smokers $29 \%$ \& $56.4 \%$ respectively when compared to non smokers, (19\%) but a significant association was not seen.

Low BMI has been recognized as a key risk factor for low BMD \& fracture. De Laet et all in a large meta analysis of 12 prospective population based cohorts of 60,000 men \& women described an inverse \& non linear relationship between BMI \& osteoporotic fracture. There was an increasing fracture risk with $\mathrm{BMI}<25 \mathrm{~kg} / \mathrm{m}^{2[14]}$. Our study also confirmed the same finding. Mean BMI in COPD patients with osteoporosis was $19.77 \mathrm{~kg} / \mathrm{m}^{2}$ .So a negative correlation was seen with BMI \& osteoporosis.

It is apparent that hyperglycemia in DM directly suppresses osteoblast-mediated bone formation, while conversely promoting osteoclast-mediated bone resorption, adipogenic differentiation of mesenchymal stem cells (also precursors of osteoblasts), and fat accumulation in the marrow cavity, all of which deteriorate bone quality and strength and increase susceptibility to fracture. ${ }^{[17]}$ Among the co-morbidities evaluated in our COPD patients, significant association was not seen with Diabetes Mellitus, only Coronary Artery Disease (CAD) was found to have a significant association with osteoporosis, likely due to the reduced physical activity .Cochrane Database demonstrated that aerobics, weight bearing \& resistance exercises are effective for improving $\mathrm{BMD}^{[16]}$

\section{Conclusion}

This study showed that COPD is a significant risk factor for osteoporosis \& as severity \& duration increases, prevalence of osteoporosis also increases. Strong association was also seen with low BMI, advanced age \& CAD. T Score was inversely proportional to Stage of COPD.
QUS estimation of BMD is an easy, cheap \& sensitive tool for early detection of osteopenia \& osteoporosis without radiation hazards, in a resource limited setting where DEXA scan is not always feasible. X-Ray spine may not detect osteopenia \& early osteoporosis.

Early detection of osteopenia/ osteoporosis in COPD patients is recommended to avoid osteoporotic fracture that deteriorates quality of life \& prognosis.

\section{Acknowledgements}

The author would like to thank Dr. Manjula V.D $\&$ the Department of Community Medicine for contribution in statistical analysis.

Study is Funded by SBMR

Disclosures: No conflict of interest

\section{References}

1. Alvar G.N Agusti 2005 ; Vol2 No4 [ATS Journals] Systemic effects of Chronic Obstructive Pulmonary Disease.

2. Alvar Agusti, All annals ATS issues $>$ Vol4/2007 Oct. Systemic effects of Chronic Obstructive Pulmonary Disease. What we know \& what we don't know.

3. Daisuke Inoue et all. International journal of Chronic Obstructive Pulmonary Disease. 2016 ;11: 637-648 . Chronic Obstructive Pulmonary Disease \& Osteoporosis: Links, Risks \& treatment challenges.

4. Jyothi Hattiholi and Gajanan S Gaude : ncbi.nim.nih.gov. Prevalence and Correlates of Osteoporosis in Chronic Obstructive Pulmonary Disease Patients in India.

5. Eugenia Quiros Roldan et al ; The Brazilian Journal of infectious diseases. $23^{\text {rd }} \quad$ Sept 2017; 21(6) 582-586. Comparison between the GOLD standard DXA with calcaneal quantitative Ultrasound based strategy (QUS) to detect osteoporosis in an HIV infected cohort. 
6. Int J Med Sci 2013 ;10( 12): 1778-1783 . Calcaneal Quantitative Ultrasound as a determinant of bone health status : What properties of bone does it reflect?

7. Juby AG, J Am Med Dir Assoc; 2004 Nov-Dec ; 5 (6): 377-81. The use of Calcaneal Ultrasound evaluation of bone mineral density in cognitively impaired seniors.

8. A. Lehouch. S Boonen, M Decramer et al; CHEST 139 (3) 2011 ; 648-657 COPD, Bone metabolism and osteoporosis

9. C Van Casteren-Messidoro, A.M Huisman et al: Netherlands, The Journal of Medicine Vol 72, No 10 , Dec2014. Quantitative Ultrasound of heel as Triage test to measure bone mineral density compared with dual energy $\mathrm{X}$ Ray absorptiometry in men with prostate $\mathrm{Ca}$ commencing with androgen deprivation therapy.

10. Eugenia Q R, Nigritella Brianese et al ; The Brazilian Journal of infectious diseases 2017; 21(6) 582-586. Comparison between the GOLD standard DXA with calcaneal quantitative Ultrasound based strategy (QUS) to detect osteoporosis in an HIV infected cohort

11. Samiha M, Abu- Bakr, Magd M Galal Eldin et al : Egyption Journal of Chest Diseases \& Tuberculosis (2014) 63, 597602. Assessment of osteoporosis in patients with Chronic obstructive pulmonary diseases .

12. A . Vrieze, MHG de Greaf et al, Osteoporos Int (2007) 18: 1197-1202 Low Bone Mineral Density in COPD patients related to worse lung function, low weight \& decreased fat-free mass.

13. Dega et al, J Pul Respair Med 2018, 8:2 Assessment of osteoporosis \& Osteopenia \& its correlation with disease severity in patients of COPD with respect to associated risk factors : A case control study in India.
14. De Laet C Kanis JA, Oden A et al: Osteoporosis Int 2005; 16: 1330-8 [Pubmed]Body mass index as a predictor of fracture risk : A Meta Analysis

15. Ward KD, Klesges RC: Calcif Tissue Int : 2001; 68(5) :259-70 [Pubmed] A metaanalysis of the effects of cigarette smoking on BMD

16. Have TE, Shea B, Davson L J et al. Cochrane database of systemic Reviews 2011: (7) doi: 10. 1002/ 14651858. c D000333. pub2 Art No: CD000333[Pubmed]

17. Wongdee K, Charoenphandhu N World J Diabetes. 2011 Mar 15;2(3):41-8. doi: 10.4239/wjd.v2.i3.41.Osteoporosis in diabetes mellitus: Possible cellular and molecular mechanisms. 